\title{
Cerebral microbleeds may contribute to cognitive decline after ischemic stroke
}

\author{
Nicolas Christ' 1 , Viola Mocke ${ }^{2}$, Wolfgang Müllges ${ }^{1}$ and Felix Fluri1
}

1Department of Neurology, University Hospital of Würzburg, Würzburg, Germany

U<

2Institute of Psychology, University of Würzburg, Würzburg, Germany

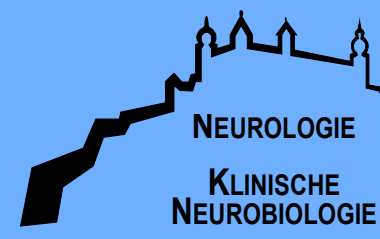

Introduction: About one third of stroke patients subsequently develop vascular cognitive decline (VCD) or vascular dementia. Whereas white matter hyperintensities were shown to predict cognitive decline following stroke, little is known about the significance of cerebral microbleeds (CMB) in the development of VCD.

Purpose: The present study aimed to investigate whether CMB contribute to VCD already in the early course after ischemic stroke, and - if so - whether distinct cognitive domains are affected more preferentially by CMB.

Methods: In a prospective cohort study, cognitive performance was examined in 33 stroke patients showing $\geq 1$ CMB on MRI. Matched for age, gender, clinical and radiological characteristics, 33 stroke survivors without CMB served as a control group. Neuropsychological testing was performed in both groups six months after the index event using the Consortium to Establish a Registry for Alzheimer's Disease (CERAD)-plus test battery. As part of the statistical analysis, we adjusted the level of significance by use of Bonferroni's correction for each cognitive domain.

\begin{tabular}{|c|c|c|c|}
\hline Group & & CMB-positive patients & Control patients \\
\hline \multirow{3}{*}{ Sex } & Male & 18 & 18 \\
\cline { 2 - 4 } & Female & 15 & 15 \\
\hline \multirow{3}{*}{ Index Event } & Stroke & 29 & 29 \\
\cline { 2 - 4 } & TIA & 4 & 4 \\
\hline \multirow{2}{*}{ Hypercholesterolemia } & Yes & 21 & 28 \\
\cline { 2 - 4 } & No & 12 & 5 \\
\hline \multirow{2}{*}{ Arterial hypertension } & Yes & 30 & 24 \\
\cline { 2 - 4 } & No & 3 & 9 \\
\hline
\end{tabular}

\begin{tabular}{|c|c|c|c|c|c|c|}
\hline \multirow{2}{*}{ Group } & \multicolumn{2}{|c|}{ CMB-positive patients } & \multicolumn{2}{|c|}{ Control patients } & \multicolumn{2}{|c|}{ Total } \\
\cline { 2 - 7 } & Mean & SD & Mean & SD & Mean & SD \\
\hline Age & 76,64 & 8,56 & 76,42 & 10,07 & 76,53 & 9,27 \\
\hline $\begin{array}{c}\text { Days } \\
\text { between } \\
\text { stroke/testing }\end{array}$ & 195,73 & 26,03 & 194,55 & 27,19 & 195,14 & 26,22 \\
\hline $\begin{array}{c}\text { NIHSS } \\
\text { admission }\end{array}$ & 3,94 & 4,34 & 3,19 & 4,62 & 3,57 & 4,46 \\
\hline $\begin{array}{c}\text { mRS } \\
\text { admission }\end{array}$ & 2,70 & 1,61 & 2,38 & 1,47 & 2,54 & 1,55 \\
\hline $\begin{array}{c}\text { Barthel-Index } \\
\text { admission }\end{array}$ & 62,97 & 28,23 & 66,52 & 30,78 & 64,77 & 29,61 \\
\hline
\end{tabular}

Results and Discussion: Stroke patients with CMB showed more severe cognitive decline in MMSE compared to the control group ( $p$ $=0,024)$. Regarding the episodic memory, CMB-positive patients reached lower scores in Word-List-Learning- $(p=0,009)$ and the Word-List-Recognition-test $(p=0,006)$, whereas the findings in Word-List-Recall-test were similar in both groups. While semantic fluency seems not to be affected, CMB-positive patients reveal a significantly impaired phonematic fluency $(p=0.037)$. Concerning the visuospatial abilities, stroke patients with $\mathrm{CMB}$ showed restricted recall of recently learned visual information. No significant differences between verum and control group were found in any test investigating the participants' executive functions. Within the semantic memory and executive functions sections, Trail-Making-Test B and phonematic fluency show the most conclusive results. Remarkably, both of these are known to depend on the participants' cognitive flexibility, which might be particularly affected by CMB. According to their location in the brain parenchyma, CMB can be classified either as lobar or deep (subcortical/ infratentorial). When only including patients showing both, lobar and deep $\mathrm{CMB}$ (mixed $\mathrm{CMB}, \mathrm{n}=20$ ), results became even more significant in certain domains. Cognitive decline intensified with an increasing number of CMB (mean pearson's $r=-0,22$ ).

\begin{tabular}{|c|c|c|c|c|c|c|c|c|c|c|c|c|c|c|}
\hline & GLOBAL & \multicolumn{4}{|c|}{ EPISODIC MEMORY } & \multicolumn{3}{|c|}{ SEMANTIC MEMORY } & \multicolumn{3}{|c|}{ VISUOSPATIAL ABILITY } & \multicolumn{3}{|c|}{ EXECUTIVE FUNCTIONS } \\
\hline Test & $\begin{array}{l}\text { Mini- } \\
\text { Mental- } \\
\text { Status }\end{array}$ & $\begin{array}{l}\text { Wordlist- } \\
\text { Learning }\end{array}$ & $\begin{array}{l}\text { Wordlist- } \\
\text { Recall }\end{array}$ & $\begin{array}{l}\text { Wordlist- } \\
\text { Savings }\end{array}$ & $\begin{array}{c}\text { Recognition } \\
\text { Discrimin- } \\
\text { ability }\end{array}$ & $\begin{array}{l}\text { Semantic } \\
\text { Fluency }\end{array}$ & $\begin{array}{l}\text { Phonematic } \\
\text { Fluency }\end{array}$ & $\begin{array}{c}\text { Boston- } \\
\text { Naming- } \\
\text { Test }\end{array}$ & $\begin{array}{l}\text { Con- } \\
\text { structional } \\
\text { Praxis }\end{array}$ & $\begin{array}{l}\text { Praxis } \\
\text { Recall }\end{array}$ & $\begin{array}{l}\text { Praxis } \\
\text { Savings }\end{array}$ & $\begin{array}{c}\text { Trail- } \\
\text { Making- } \\
\text { Test A }\end{array}$ & $\begin{array}{l}\text { Trail- } \\
\text { Making- } \\
\text { Test B }\end{array}$ & $\begin{array}{l}\text { Trail- } \\
\text { Making- } \\
\text { Test B/A }\end{array}$ \\
\hline \begin{tabular}{|c} 
Level of \\
significance \\
after \\
adjustment
\end{tabular} & 0,050 & 0,012 & 0,012 & 0,012 & 0,012 & 0,017 & 0,017 & 0,017 & 0,017 & 0,017 & 0,017 & 0,017 & 0,017 & 0,017 \\
\hline $\mathrm{p}$-value & 0,024 & 0,009 & 0,028 & 0,193 & 0,006 & 0,296 & 0,007 & 0,039 & 0,216 & 0,004 & 0,003 & 0,296 & 0,046 & 0,173 \\
\hline $\begin{array}{c}\text { Mixed CMB } \\
\text { p-value }\end{array}$ & 0,048 & 0,016 & 0,001 & 0,097 & 0,001 & 0,129 & 0,001 & 0,070 & 0,246 & 0,010 & 0,010 & 0,056 & 0,025 & 0,263 \\
\hline
\end{tabular}

results of neuropsychological investigation

Conclusion: Six months after stroke, cognitive abilities are more severely affected if patients are CMB-positive. Stroke survivors with $\mathrm{CMB}$ might more often experience impaired episodic memory and phonematic fluency. Besides, they show deficits in recollecting visual information. However, further studies are needed to confirm these findings. 